R. F. Shamoyan, S. M. Kurilenko

\title{
ON EXTREMAL PROBLEMS IN TUBULAR DOMAINS OVER SYMMETRIC CONES ${ }^{1}$
}

\begin{abstract}
We prove new sharp distance theorems for analytic spaces in tube domains over symmetric cones extending our previous results in this direction
\end{abstract}

Key words: Distance estimates, analytic function, tube domains.

2010 Mathematical Subject Classification: 42B15, 42B30.

\section{Introduction}

In this note we obtain several sharp distance estimates in spaces of analytic spaces in tube domains over symmetric cones.

This line of investigation can be considered as a continuation of previous papers [1], [2] and [3].

These new results are contained in the second and third section of this note. We remark that for the first time in literature we consider this extremal problem related to distance estimates in spaces of analytic functions on tube domains over symmetric cones. The first section contains required preliminaries on analysis on symmetric cones.

In one dimensional tubular domain which is upperhalfspace $C_{+}$(see [4]) our theorems are not new and they were obtained recently in [5].

Moreover, arguments in proofs we provided below are similar to those we have in one dimension and the base of proof is again the so-called Bergman reproducing formula, but in the tubular domain over symmetric cone. (see, for example, [4] for this integral representation).

We shortly remind the history of this problem.

\footnotetext{
${ }^{1}$ This work was supported by the Russian Foundation for Basic Research (project No. 13-353 01-97508) and by the Ministry of Education and Science of the Russian Federation (project No.1.1704.2014K).

(C) Shamoyan R. F., Kurilenko S. M., 2014
} 
After the appearance of [6] various papers appeared where arguments which can be seen in [6] were extended and modifyied in various directions $[1-3]$.

In particular in mentioned papers various new results on distances for analytic function spaces in higher dimension (unit ball and polydisk) were obtained. Namely new results for large scales of analytic mixed norm spaces in higher dimension were proved.

Later several new sharp results for harmonic functions of several variables in the unit ball and upperhalfplane of Euclidean space were also obtained, see, for example, [1] and references there.

We mention separatly [5] and [7] where the case of higher dimension was considered in special cases of analytic spaces on subframe and new analogues results in the context of bounded strictly pseudoconvex domains with smooth boundary were also provided.

The classical Bergman representation formula in various forms and in various domains serves as a base in all these papers in proofs of main results.

We would like to note also the recent results, obtained by Wen $\mathrm{Xu}$ in [8], repeating arguments of Ruhan Zhao in the unit ball, on distances from Bloch functions to some Mobius invariant function spaces in one and higher dimension in a relatively direct way.

Probably for the first time in literature these extremal problems are connected with distances in analytic spaces appeared before in [9] and in [10] where this problem was formulated and certain cases connected with spaces of bounded analytic functions in the unit disk were considered.

These results were mentioned [11], some other results on distance problems in BMOA spaces may be found also in [12].

Various other extremal problems in analytic function spaces also were considered before in various papers, see for example [13-16].

In those papers other results around this topic and some applications of certain extremal problems may also be found.

The goal of this note is to develop further some ideas from our recent mentioned papers and present a new sharp theorem in a tube domain over symmetric cones.

It is worth nothing note that in the case of upper halfplane of complex plane $C$ which is a tube domain in one dimension such results have already been obtained previously by author [5]. Recently authors obtained a series of sharp results for distances in analytic function spaces on products of various domains (Siegel domains of second type, tubular domains). For 
formulation of our results we will need various standard definitions from the theory of tube domains over symmetric cones (see [4], [17-19]).

Let $T_{\Omega}=V+i \Omega$ be the tube domain over an irreducible symmetric cone $\Omega$ in the complexification $V^{\mathbb{C}}$ of an $n$-dimensional Euclidean space $V$. $\mathcal{H}\left(T_{\Omega}\right)$ denotes the space of all holomorphic functions on $T_{\Omega}$. Following the notation of [18] and [4] we denote the rank of the cone $\Omega$ by $r$ and by $\Delta$ the determinant function on $V$.

Letting $V=\mathbb{R}^{n}$, we have as an example of a symmetric cone on $\mathbb{R}^{n}$ the Lorentz cone $\Lambda_{n}$ which is a rank 2 cone defined for $n \geq 3$ by

$$
\Lambda_{n}=\left\{y \in \mathbb{R}^{n}: y_{1}^{2}-\cdots-y_{n}^{2}>0, y_{1}>0\right\} .
$$

The determinant function in this case is given by the Lorentz form

$$
\Delta(y)=y_{1}^{2}-\cdots-y_{n}^{2}
$$

(see for example [4]).

Let us introduce some convenient notations regarding multi-indices.

If $t=\left(t_{1}, \ldots, t_{r}\right)$, then $t^{\star}=\left(t_{r}, \ldots, t_{1}\right)$ and, for $a \in \mathbb{R}, t+a=$ $=\left(t_{1}+a, \ldots, t_{r}+a\right)$. Also, if $t, k \in \mathbb{R}^{r}$, then $t<k$ means $t_{j}<k_{j}$ for all $1 \leq j \leq r$.

We are going to use the following multi-index

$$
g_{0}=\left((j-1) \frac{d}{2}\right)_{1 \leq j \leq r}, \quad \text { where } \quad(r-1) \frac{d}{2}=\frac{n}{r}-1
$$

For $\tau \in \mathbb{R}_{+}$and the associated determinant function $\Delta(x)[4]$ we set

$$
A_{\tau}^{\infty}\left(T_{\Omega}\right)=\left\{F \in \mathcal{H}\left(T_{\Omega}\right):\|F\|_{A_{\tau}^{\infty}}=\sup _{x+i y \in T_{\Omega}}|F(x+i y)| \Delta^{\tau}(y)<\infty\right\} .
$$

It can be checked that this is a Banach space. Below we denote by $\Delta_{s}$ the generalized power function [18], [4].

For $1 \leq p, q<+\infty$ and $\nu \in \mathbb{R}$, and $\nu>\frac{n}{r}-1$ we denote by $A_{\nu}^{p, q}\left(T_{\Omega}\right)$ the mixed-norm weighted Bergman space consisting of analytic functions $f$ in $T_{\Omega}$ such that

$$
\|F\|_{A_{\nu}^{p, q}}=\left(\int_{\Omega}\left(\int_{V}|F(x+i y)|^{p} d x\right)^{q / p} \Delta^{\nu}(y) \frac{d y}{\Delta(y)^{n / r}}\right)^{1 / q}<\infty .
$$


This is a Banach space.

Replacing above A by L we will get as usual the corresponding larger space of all measurable functions in tube over symmetric cone with the same quazinorm (see [18], [19]).

It is known that the $A_{\nu}^{p, q}\left(T_{\Omega}\right)$ space is nontrivial if and only if $\nu>$ $>\frac{n}{r}-1,($ see $[17],[4])$.

When $p=q$ we write (see [4])

$$
A_{\nu}^{p, q}\left(T_{\Omega}\right)=A_{\nu}^{p}\left(T_{\Omega}\right) .
$$

This is the classical weighted Bergman space with usual modification when $p=\infty$.

The (weighted) Bergman projection $P_{\nu}$ is the orthogonal projection from the Hilbert space $L_{\nu}^{2}\left(T_{\Omega}\right)$ onto its closed subspace $A_{\nu}^{2}\left(T_{\Omega}\right)$ and it is given by the following integral formula (see [4])

$$
P_{\nu} f(z)=C_{\nu} \int_{T_{\Omega}} B_{\nu}(z, w) f(w) d V_{\nu}(w)
$$

where

$$
B_{\nu}(z, w)=C_{\nu} \Delta^{-\left(\nu+\frac{n}{r}\right)}((z-\bar{w}) / i)
$$

is the Bergman reproducing kernel for $A_{\nu}^{2}\left(T_{\Omega}\right)$ (see [18], [4]).

Here we used the notation $d V_{\nu}(w)=\Delta^{\nu-\frac{n}{r}}(v) d u d v$. We denote by $d V(w)$ or $d v(w)$ the Lebegues measure on tubular domain over symmetric cone. Below and here we use constantly the following notations $w=$ $=u+i v \in T_{\Omega}$ and also $z=x+i y \in T_{\Omega}$.

Hence for any analytic function from $A_{\nu}^{2}\left(T_{\Omega}\right)$ the following integral formula is valid (see also [4])

$$
f(z)=C_{\nu} \int_{T_{\Omega}} B_{\nu}(z, w) f(w) d V_{\nu}(w) .
$$

In this case sometimes below we say simply that the $f$ function allows Bergman representation via Bergman kernel with $\nu$ index.

Note that these assertions have direct copies in simpler cases of analytic function spaces in unit disk, polydisk, unit ball, upperhalfspace $C_{+}$ and in spaces of harmonic functions in the unit ball or upperhalfspace of Euclidean space $R^{n}$ These classical facts are well-known and can be found, for example, in [20] and in some items from references there.

Above and throughout the paper we write $C$ (sometimes with indexes) to denote positive constants which might be different each time we see 
them (and even in a chain of inequalities), but are independent of the functions or variables being discussed.

In this paper we will also need a pointwise estimate for the Bergman projection of functions in $L^{p, q}\left(T_{\Omega}\right)$, defined by integral formula (2), when this projection has sense. Note that such estimates in simpler cases of unit disk, unit ball and polydisk are well-known (see [20]).

Let us first recall the following known basic integrability properties for the determinant function, which appeared already above in definitions.

Lemma 1. Let $\alpha \in \mathbb{C}^{r}$ and $y \in \Omega$.

1) The integral

$$
J_{\alpha}(y)=\int_{\mathbb{R}^{n}}\left|\Delta_{-\alpha}\left(\frac{x+i y}{i}\right)\right| d x
$$

converges if and only if Re $\alpha>g_{0}^{*}+\frac{n}{r}$. In this case $J_{\alpha}(y)=C_{\alpha}\left|\Delta_{-\alpha+n / r}(y)\right|$.

2) For any multi-indices $s$ and $\beta$ from $C^{r}$ and $t \in \Omega$ the function

$$
y \mapsto \Delta_{\beta}(y+t) \Delta_{s}(y)
$$

belongs to $L^{1}\left(\Omega, \frac{d y}{\Delta^{n / r}(y)}\right)$ if and only if $\operatorname{Re}(s)>g_{0}$ and $\operatorname{Re}(s+\beta)<-g_{0}^{*}$. In this case we have

$$
\int_{\Omega} \Delta_{\beta}(y+t) \Delta_{s}(y) \frac{d y}{\Delta^{n / r}(y)}=C_{\beta, s} \Delta_{s+\beta}(t) .
$$

We refer to Corollary 2.18 and Corollary 2.19 of [17] for the proof of the above lemma or [4].

As a corollary of one dimensional versions of these estimates (see, for example, [19] Theorem 3.9) we obtain the following vital estimate (A) which we will use in proof of our main result.

$$
\int_{T_{\Omega}} \Delta^{\beta}(y)\left|B_{\alpha+\beta+\frac{n}{r}}(z, w)\right| d V(z) \leq C \Delta^{-\alpha}(v)
$$

$\beta>-1, \alpha>\frac{n}{r}-1, z=x+i y, w=u+i v($ see $[19])$.

Let $\tau$ be the set of all triples $(p, q, \nu)$ such that $1 \leq p, q<\infty, \nu>\frac{n}{r}-1$.

The following vital pointwise estimate can be found, for example, in $[4]$. 
Lemma 2. Suppose $(p, q, \nu) \in \tau$. Then

$$
\left|P_{\nu} f(z)\right| \leq c_{p, q, r, \nu, n} \Delta^{-\frac{\nu}{q}-\frac{n}{r p}}(\operatorname{Im} z)\|f\|_{A_{\nu}^{p, q} .}
$$

Proof. This is a consequence of the lemma formulated above and Hölder's inequality (see [4]).

Let $H^{p}\left(T_{\Omega}\right)$ be standard Hardy space in tubular domain over symmetric cone. Let further $H_{\alpha}^{p}\left(T_{\Omega}\right)$ be weighted Hardy class with $\Delta^{\alpha}(y)$ weight, see $[4]$.

These are spaces with finite norm

$$
\sup _{y \in \Omega} \int_{R^{n}}|f(\tau+i y)|^{p} d \tau \Delta^{p \alpha}(y)
$$

$1 \leq p<\infty, \alpha \in R$.

Let $1 \leq p<\infty, 1 \leq q<\infty$. Let also $q \leq s$. Then (see [4]) $A_{\nu}^{p, q} \subset H_{\beta}^{s}$, where $\nu>\frac{n}{r}-1$ and where $\beta=\frac{\nu}{q}+\frac{n}{r p}-\frac{n}{r s}$. For $p=q=s$ this embedding with appropriate estimate is taking obviously a very simple form (see [4] proposition 3.5) and the distance problem here can be easily posed again obviously in general case and in mentioned simple case. Note that for analytic and harmonic function spaces it was posed and solved in [1-3]. Repeating arguments from these papers, an analogous result can also be obtained, using approaches we provided above. This will be done in section 2 .

We define $\tilde{H}_{s}^{p}, 1 \leq p<\infty, s \in R$ as a subset of $H_{s}^{p}$ so that for each $\mathrm{f}$ function from that subset the Bergman representation formula with large enough $\alpha_{0}$ index is valid.

\section{$\S 1$. New estimates for distances $\operatorname{dist}_{A_{\alpha}^{\infty}}\left(f, A_{t}^{p}\right)$ in analytic function spaces in tube domains over symmetric cones}

In this paper we restrict ourselves to a irreducible symmetric cone $\Omega$ in the Euclidean vector space $R^{n}$ of dimension $\mathrm{n}$, endowed with an inner product for which the cone $\Omega$ is self dual. We denote by $T_{\Omega}=R^{n}+i \Omega$ the corresponding tube domain in $C^{n}$.

This section is devoted to formulations and proofs of some of our main results of this paper. As previously in case of an analytic functions in a unit disk, a polydisk, a unit ball, and an upperhalfspace $C_{+}$and in the 
case of spaces of harmonic functions in Euclidean space [1-3], [5-7] the role of the Bergman representation formula is crucial in these issues and our proof is heavily based on it.

As it is known a variant of Bergman representation formula is available also in Bergman-type analytic function spaces in tubular domains over symmetric cones and this well-known fact (see [4], [17-19]), which is also crucial in various problems in analytic function spaces in tubular domains (see [4] and various references there) and is used also in our proofs below.

The following result can be found in [19] (section 4).

For all $1<p<\infty$ and $1<q<\infty$ and for all $\frac{n}{r} \leq p_{1}$, where $\frac{1}{p_{1}}+\frac{1}{p}=1$, and $\frac{n}{r}-1<\nu$, and for all functions $f$ from $A_{\nu}^{p, q}$ and for all $n / r-1<\alpha$ the Bergman representation formula with $\alpha$ index or with the Bergman kernel $B_{\alpha}(z, w)$ is valid.

We remark that this result is a particular case of a more general assertion for analytic mixed norm $A_{\nu}^{p, q}$ classes (see [19]) which means that our main result below admits also some extensions, even to mixed norm spaces which we defined above, this will be discussed below at the end and in our next paper which is in preparation.

We will also need for our proofs the following important fact on integral representations (see [21]). Let $\nu>\frac{n}{r}-1, \alpha>\frac{n}{r}-1$, then for all functions from $A_{\alpha}^{\infty}$ the integral representations of Bergman with Bergman kernel $B_{\alpha+\nu}(z, w)$ (with $\alpha+\nu$ index) is valid.

We note also that by Lemma 2 we have

$$
|f(x+i y)| \Delta^{\frac{n}{r p}+\frac{\nu}{q}}(y) \leq c_{p, q, r, \nu}\|f\|_{A_{\nu}^{p, q}}
$$

$x+i y \in T_{\Omega},(p, q, \nu) \in \tau$. This means that we have a continuous embedding $A_{\nu}^{p} \hookrightarrow A_{\frac{n}{r p}+\frac{\nu}{p}}^{\infty}$ for $(p, p, \nu) \in \tau$ and this naturally leads to a problem of estimating

$$
\operatorname{dist}_{A_{\frac{n}{r p}+\frac{\nu}{p}}^{\infty}}\left(f, A_{\nu}^{p}\right)
$$

for a given $f \in A_{\frac{n}{r p}+\frac{\nu}{p}}^{\infty}$.

This problem is solved in our next theorem below, which is the main result of this section. Let us set, for $f \in \mathcal{H}\left(T_{\Omega}\right), s \in \mathbb{R}$ and $\epsilon>0$ :

$$
V_{\epsilon, s}(f)=\left\{x+i y \in T_{\Omega}:|f(x+i y)| \Delta^{s}(y) \geq \epsilon\right\}
$$


Let also $w=u+i v \in T_{\Omega}, z=x+i y \in T_{\Omega}$. We denote by $N_{1}$ and by $N_{2}$ two sets - the first one is $V_{\epsilon, s}(f)$, the other one is the set of all those points, which are in tubular domain $T_{\Omega}$, but not in $N_{1}$.

Theorem 1. Let $1<p<\infty, \nu>p\left(\frac{n}{r}-1\right), \beta>t+\frac{n}{r}-1, t=\frac{1}{p}\left(\nu+\frac{n}{r}\right)$. Set, for $f \in A_{\frac{n}{r p}+\frac{\nu}{p}}^{\infty}$ :

$$
\begin{gathered}
l_{1}(f)=\operatorname{dist}_{A_{\frac{n}{r p}+\frac{\nu}{p}}^{\infty}}\left(f, A_{\nu}^{p}\right) \\
l_{2}(f)=\inf \left\{\epsilon>0: \int_{T_{\Omega}}\left(\int_{V_{\epsilon, t}(f)} \frac{\Delta^{\beta-t-\frac{n}{r}}(v) d u d v}{\Delta^{\beta+\frac{n}{r}} \frac{(z-\bar{w})}{i}}\right)^{p} \Delta^{\nu-\frac{n}{r}}(y) d x d y<\infty\right\}
\end{gathered}
$$

Then there is a positive number $\beta_{0}$, so that for all $\beta>\beta_{0}$ we have $l_{1}(f) \asymp l_{2}(f)$.

Proof. We will use for our proofs the following observation (see [21]). Let $\nu>\frac{n}{r}-1, \tau>\frac{n}{r}-1$, then for all functions from $A_{\tau}^{\infty}$ the integral represenations of Bergman with Bergman kernel

$$
B_{\tau+\nu}(z, w)
$$

is valid.

We denote below the double integral which appeared in formulation by $G(f)$ and we will show first that $l_{1}(f) \leq C l_{2}(f)$. We assume now that $l_{2}(f)$ is finite.

We use the Bergman representation formula which we provided above, namely (3), and using conditions on parameters we have the following equalities.

First we have obviously by remark, with which we started this proof, that for large enough $\beta$

$$
\begin{gathered}
f(z)=C_{\nu} \int_{T_{\Omega}} B_{\beta}(z, w) f(w) d V_{\beta}(w)=f_{1}(z)+f_{2}(z), \\
f_{1}(z)=C_{\nu} \int_{N_{2}} B_{\beta}(z, w) f(w) d V_{\beta}(w), \\
f_{2}(z)=C_{\nu} \int_{N_{1}} B_{\beta}(z, w) f(w) d V_{\beta}(w) .
\end{gathered}
$$

Then we estimate both functions separately using lemmas provided above and following some arguments we provided in one dimensional case 
that is the case of upper-half space $C_{+}$(see [5]). Here our arguments are sketchy since they are parallel to arguments from [5]. Using definitions of $N_{1}$ and $N_{2}$, proposed above, after some calculations, following arguments from [5] and using estimation (A), we will have immediately

$$
f_{1} \in A_{\frac{n}{r p}+\frac{\nu}{p}}^{\infty}
$$

and

$$
f_{2} \in A_{\nu}^{p}
$$

We easily note that the last inclusion follows directly from the fact that $l_{2}$ is finite.

Moreover, it can be easily seen that the norm of $f_{1}$ can be estimated from above by $C \epsilon$, where for some positive constant $C$ (see [5]), since obviously

$$
\sup _{N_{2}}|f(w)| \Delta^{t}(v) \leq \epsilon
$$

Note that this last fact follows directly from the definition of $N_{2}$ set and estimates in lemma above which leads to the following inequality which was denoted by us as (A) (see also [19]).

$$
\int_{T_{\Omega}} \Delta^{-t}(y)\left|B_{\beta}(z, w)\right| d V_{\beta}(z) \leq C \Delta^{-t}(v),
$$

$z=x+i y, w=u+i v$, for all $\beta$ so that $\beta>\beta_{0}$, for some large enough fixed $\beta_{0}$ which depends on $n, r, \nu, p$ and for $t=\left(\frac{1}{p}\right)\left(\nu+\frac{n}{r}\right)$ and $\nu>p\left(\frac{n}{r}-1\right)$ (see [19] Theorem 3.9).

This gives immediately one part of our theorem. Indeed, we have obviously

$$
l_{1} \leq C_{2}\left\|f-f_{2}\right\|_{A_{t}^{\infty}}=C_{3}\left\|f_{1}\right\|_{A_{t}^{\infty}} \leq C_{4} \epsilon .
$$

It remains to prove that $l_{2} \leq l_{1}$. Let us assume that $l_{1}<l_{2}$. Then there are two numbers $\epsilon$ and $\epsilon_{1}$, both positive such that there exists $f_{\epsilon_{1}}$, so that this function is in $A_{\nu}^{p}$ and $\epsilon>\epsilon_{1}$ and also the following conditions holds

$$
\left\|f-f_{\epsilon_{1}}\right\|_{A_{t}^{\infty}} \leq \epsilon_{1}
$$

and $G(f)=\infty$, where $G$ is a double integral in formulation of theorem in $l_{2}($ see $(8))$. 
Next from

$$
\left\|f-f_{\epsilon_{1}}\right\|_{A_{t}^{\infty}} \leq \epsilon_{1}
$$

we have the following two estimates, the second one is a direct corollary of the first one. First we have for $z=x+i y$

$$
\left(\epsilon-\epsilon_{1}\right) \tau_{V_{\epsilon, t}}(z) \Delta^{-t}(y) \leq C\left|f_{\epsilon_{1}}(z)\right|,
$$

where $\tau_{V_{\epsilon, t}}(z)$ is a characteristic function of $V=V_{\epsilon, t}(f)$ set we have defined above.

And from the last estimation we have directly multiplying both sides by Bergman kernel $B_{\beta}(z, w)$ and integrating by tube $T_{\Omega}$ both sides with the measure $d V_{\beta}$

$$
G(f) \leq C \int_{T_{\Omega}}\left(L\left(f_{\epsilon_{1}}\right)\right)^{p} \Delta^{\nu-\frac{n}{r}}(y) d y d x
$$

where

$$
L=L\left(f_{\epsilon_{1}}, z\right)
$$

and

$$
L\left(f_{\epsilon_{1}}, z\right)=\int_{T_{\Omega}}\left|f_{\epsilon_{1}}(w)\right|\left|B_{\beta}(z, w)\right| d V_{\beta}(w) .
$$

Denote this expression by $I$. Put $\beta+\frac{n}{r}=k_{1}+k_{2}$, where $k_{1}=\beta-\frac{n}{r}-\mu$, $k_{2}=\mu+2 \frac{n}{r}\left(\frac{1}{p}+\frac{1}{p_{1}}\right)$.

By classical Holder inequality with $p$ and $p_{1}, p^{-1}+p_{1}^{-1}=1$ we obviously have $I^{p} \leq C I_{1} I_{2}$, where

$$
\begin{gathered}
I_{1}(f)=\int_{T_{\Omega}}\left|f_{1}(z)\right|^{p}\left|\Delta^{s}((z-\bar{w}) / i)\right| \Delta^{\left(\beta-\frac{n}{r}\right) p}(y) d x d y, \\
I_{2}^{\frac{p_{1}}{p}}=\int_{T_{\Omega}}\left|\Delta^{v}((z-\bar{w}) / i)\right| d x d y
\end{gathered}
$$

where $f_{1}=f_{\epsilon_{1}}$ and $s=\mu p-2 \frac{n}{r}-\beta p+p \frac{n}{r}, v=-2 \frac{n}{r}-\mu p_{1}$.

Choosing finally $\mu$, so that the estimate (A) can be used twice above and finally making some additional calculations we will get what we need.

Note here we have to use the fact that

$$
\nu>p\left(\frac{n}{r}-1\right)
$$

which was given in formulation of our theorem. 
Hence we have now,

$$
\begin{gathered}
\int_{T_{\Omega}}\left(\int_{T_{\Omega}}\left|f_{\epsilon_{1}}(z)\right| B_{\beta}(z, w) \mid d V_{\beta}(z)\right)^{p} \Delta^{\nu-\frac{n}{r}}(v) d V(w) \leq C\left\|f_{\epsilon_{1}}\right\|_{A_{\nu}^{p}}^{p} \\
G(f) \leq C\left\|f_{\epsilon_{1}}\right\|_{A_{\nu}^{p}},
\end{gathered}
$$

but we also have

$$
f_{\epsilon_{1}} \in A_{\nu}^{p} .
$$

This will give as a contradiction with our assumption above that $G(f)=\infty$. So we proved the estimate which we wanted to prove. The proof of our first theorem is now complete.

Finally, we add some vital remarks. Similar results are also true for certain analytic Besov spaces namely $B_{\nu}^{p, q}$ classes in tube domains over symmetric cones. To get such result for these classes we have to repeat arguments in proofs of theorems above and use at final step in proofs the embedding theorems which connect them directly with Bergman spaces (see [17], [22]). For almost all facts we mention below we refer the reader, for example, to [22]. We refer the reader to the definition of analytic Besov $B_{\nu}^{p, q}$ spaces to [22]. In the following result we use the notation $q_{\nu, p}=\left(\min \left(p, p_{1}\right)\right) q_{\nu}, q_{\nu}=\frac{\nu+\frac{n}{r}-1}{\frac{n}{r}-1}, \frac{1}{p}+\frac{1}{p_{1}}=1$. The problem on distances in analytic Besov spaces can still be posed since

$$
B_{\nu}^{p, q} \subset A_{\frac{n}{r p}+\frac{\nu}{q}}^{\infty}
$$

for $\nu>0,1 \leq p<\infty, 1<q<Q_{\nu, p}$, for certain $Q_{\nu, p}, Q_{\nu, p}=\frac{\nu+\frac{n}{r}-1}{L}$, $L=\max \left(0, \frac{n}{r p_{1}}-1\right)$, for $\frac{n}{r} \leq p_{1}$ we put $Q_{\nu, p}=\infty$, [19]. Note that the following estimate is true $q_{\nu, p} \leq Q_{\nu, p}$. Various projection theorems in analytic Besov spaces in tube domains over symmetric cones have been well studied recently in [22]. Note $B_{0}^{2}=H^{2}$, where $H^{p}$ is analytic Hardy space in tube domain over symmetric cone.

We remark (see [22]) that the $A_{\nu}^{p, q}$ class for values of parameters which we consider is a dense subspace of $B_{\nu}^{p, q}$ and hence the Bergman representation formula is valid for all functions from this Besov class [22]. Note also that the Bergman representation formula with Bergman kernel with index $\alpha$ is valid for all functions from $A_{\nu}^{p, q}$ for $\alpha>\frac{n}{r}-1$ and for $1 \leq p<\infty$, and $1 \leq q<Q_{\nu, p}$ (see for example [19] and references there). We can hence formulate our theorem via analytic Besov spaces. The restriction 
on $p$ from formulation of previous theorems will be replaced by new restrictions on $p$ via $Q_{\nu, p}$ and $q_{\nu, p}$ which allows to use embeddings we need, connecting analytic Besov and Bergman classes (see [19], [22]) and which also allows to pose a problem on distances in analytic Besov class.

\section{$\S 2$. On new sharp estimates for $\operatorname{dist}_{H_{s}^{v}}\left(f, A_{\nu}^{v}\right)$ and new related estimates for mixed norm spaces}

In this section we consider extremal problems based on embeddings connecting analytic Hardy and Bergman spaces in tubular domains.

$$
\text { Let } L_{s, \varepsilon, v}(f)=\left\{y \in \Omega:\left(\int_{R^{n}}|f(x+i y)|^{v} d x\right)^{\frac{1}{v}}\left(\Delta^{s}(y)\right) \geq \varepsilon\right\}
$$

for $\varepsilon>0, s>0, v>0$ and for an analytic function in $T_{\Omega}$ tube.

Note, as we mentioned above, that $A_{\nu}^{v}$ is embedded in $H_{s}^{v}$ for $s=\frac{\nu}{v}$ so we can pose a distance problem, $1 \leq v<\infty$.

Theorem 2. Let $s=\frac{\nu}{v}, 1 \leq v<\infty, s \in R, \nu>v\left(\frac{n}{r}-1\right)$. Let $f \in$ $\in \tilde{H}_{s}^{v}$. Then we have $l_{1}(f) \asymp l_{2}(f)$; where $l_{1}(f)=\operatorname{dist}_{H_{s}^{v}}\left(f, A_{\nu}^{v}\right) ; \frac{n}{r}<v^{\prime}$; $\frac{1}{v}+\frac{1}{v^{\prime}}=1$.

$$
l_{2}(f)=\inf \left\{\varepsilon>0: \int_{\Omega}\left(\tau_{L_{\varepsilon, s, v}(f)}(y)\right)\left(\Delta^{-\frac{n}{r}}(y)\right) d y<\infty\right\} .
$$

Proof. (of one part of theorem 2).

First we prove that $l_{1}(f) \geq l_{2}(f)$ in a general form for $A_{s p}^{v, p}$ spaces. Let us assume that $l_{1}<l_{2}$. Then there are two numbers $\varepsilon, \varepsilon_{1}, \varepsilon>0, \varepsilon_{1}>0$ such that $f_{\varepsilon_{1}} \in A_{\nu}^{v, p} ; \varepsilon>\varepsilon_{1},\left\|f-f_{\varepsilon_{1}}\right\|_{H_{s}^{v}} \leq \varepsilon_{1}$ and

$$
\int_{\Omega}\left(\tau_{L_{\varepsilon, s}(f)}(y)\right)\left(\Delta^{-\frac{n}{r}}(y)\right) d y=\infty .
$$

We consider general case now. Hence we have

$$
\begin{gathered}
\left(\int_{R^{n}}\left|f_{\varepsilon_{1}}(\tau+i y)\right|^{v} d \tau\right)^{\frac{1}{v}}\left(\Delta^{s}(y)\right) \geq \Delta^{s}(y)\left(\int_{R^{n}}|f(\tau+i y)|^{v} d \tau\right)^{\frac{1}{v}}- \\
-\sup _{y \in \Omega}\left[\Delta^{s}(y)\right]\left(\int_{R^{n}}\left|f(\tau+i y)-f_{\varepsilon_{1}}(\tau+i y)\right|^{v} d \tau\right)^{\frac{1}{v}} \geq \\
\geq\left(\Delta^{s}(y)\right)\left(\int_{R^{n}}|f(\tau+i y)|^{v} d \tau\right)^{\frac{1}{v}}-\varepsilon_{1} .
\end{gathered}
$$


Hence we have now

$$
\begin{gathered}
\left(\varepsilon-\varepsilon_{1}\right)^{p} \int_{\Omega}(\Delta(y))^{\tilde{\alpha}}\left[\lambda_{\tilde{L}_{\varepsilon, s}}(f)(y)\right] d y \leq \\
\leq c \int_{\Omega}\left(\int_{R^{n}}\left|f_{\varepsilon_{1}}(\tau+i y)\right|^{v} d \tau\right)^{\frac{p}{v}} \Delta^{s p}(y) \Delta^{\alpha-\frac{n}{r}}(y) d y
\end{gathered}
$$

where $\tilde{\alpha}=-\frac{n}{r}+\alpha$ for any $\alpha \geq 0$. We have a contradiction.

We actually proved the following theorem:

Theorem 3. Let $s p>n / r-1 ; p \leq v ; v \in[1, \infty), p \in[1, \infty), s \in R$, $f \in \tilde{H}_{s}^{v}$, Then

$$
\begin{gathered}
\operatorname{dist}_{H_{s}^{v}}\left(f, A_{s p}^{v, p}\right) \geq \inf \left\{\varepsilon>0: \int_{\Omega} \tau_{\tilde{L}_{\varepsilon, s, v}}(f)(y) \times\left(\Delta^{-n / r}(y)\right) d y<\infty\right\} ; \\
L_{\varepsilon, s, v}(f)=\left\{y \in \Omega:\left(\int_{R^{n}}|f(x+i y)|^{v} d x\right)^{\frac{1}{v}} \Delta^{s}(y) \geq \varepsilon\right\} .
\end{gathered}
$$

Remark 1. Note that for $v=p=1$, this result is known (see [23]).

We will return to $p=v$ case and below we'll show sharp theorem 2 . Note for that we have to prove only the reverse part since one part follows from theorem 3 .

Note again that

$$
A_{\nu}^{p, q}=\left\{f \in H\left(T_{\Omega}\right):\left(\int_{\Omega}\left(\int_{R^{n}}|f(x+i y)|^{p} d x\right)^{\frac{q}{p}}\left(\Delta^{\nu-n / r}(y)\right) d y\right)^{\frac{1}{q}}<\infty\right\} ;
$$

$1 \leq p, q<\infty, \nu>\frac{n}{r}-1$.

Note that the base of problem is the embedding $A_{\nu}^{p, v} \subset H_{\beta}^{s} ; 1 \leq p<$ $<\infty ; 1 \leq v<\infty ; v \leq s ; \nu>\frac{n}{r}-1 ; \beta=\frac{\nu}{v}+n\left(\frac{1}{r p}-\frac{1}{r s}\right)$ (see [23], [4]).

Note if $v=p, s=1$ then we have

1) $A_{\nu}^{p}=A_{\nu}^{p, p} \subset H_{\beta}^{s} ; 1 \leq p<\infty ; p \leq s ; \nu>\frac{n}{r}-1 ; \beta=\frac{\nu}{p}+\frac{n}{r}\left(\frac{1}{p}-\frac{1}{s}\right)$;

2) If $v=s=1$ we have then $A_{\nu}^{p, 1} \subset H_{\beta}^{1} ; 1 \leq p<\infty ; \nu>\frac{n}{r}-1$; $\beta=\nu+\frac{n}{r}\left(\frac{1}{p}-1\right)$

3) If $p=s, \nu=\beta, A_{\beta}^{p, 1} \subset H_{\beta}^{p} ; 1 \leq p<\infty, \nu>\frac{n}{r}-1$; 
4) If $p=1, v=s, A_{\nu}^{1, s} \subset H_{\beta}^{s} ; s \geq 1 ; \nu>\frac{n}{r}-1 ; \beta=\frac{\nu}{s}+\frac{n}{r}(1-1 / s)$.

Each embedding poses dist problem which was partially solved in theorem 3 by us.

The mentioned four cases are of main interest for this paper and we will focus on them now trying to prove a new result in all cases separately, note that for $p=1 ; s=1$ all cases coincide and the sharp theorem was already proved before in [23].

\section{Theorem 4.}

1) Let $1 \leq p<\infty ; \nu>\frac{n}{r}-1 ; \beta=\nu+(n / r)(1 / p-1)$. Then let $f \in \tilde{H}_{\beta}^{1}$, then $l_{1}(f) \leq l_{2}(f)$, where $l_{1}(f)=\operatorname{dist}_{\tilde{H}_{\beta}^{1}}\left(f, A_{\nu}^{p, 1}\right)$;

$$
l_{2}(f)=\inf \left\{\varepsilon>0: \int_{\Omega}\left(\tau_{L_{\varepsilon, \beta, 1}(f)}(y)\right)\left(\Delta^{-\frac{n}{r}}(y)\right) d y<\infty\right\} .
$$

2) Let $v \geq 1 ; \nu>\frac{n}{r}-1$. Let $f \in H_{s}^{v}$ then $l_{1}(f) \geq l_{2}(f)$, where $l_{1}(f)=\operatorname{dist}_{H_{s}^{v}}\left(f, A_{\nu}^{1, v}\right)$;

$$
l_{2}(f)=\inf \left\{\varepsilon>0: \int_{\Omega}\left(\tau_{L_{\varepsilon, s, v}(f)}(y)\right)\left(\Delta^{-\frac{n}{r}}(y)\right) d y<\infty\right\},
$$

$s=\frac{\nu}{v}+\frac{n}{r}\left(1-\frac{1}{v}\right)$.

3) Let $f \in \tilde{H}_{s}^{v}, s=\nu / v, v \geq 1, \frac{\nu}{v}-\frac{n}{r} \geq 1, \nu>\frac{n}{r}-1$. Then $l_{1}(f)$ is equivalent to $l_{2}(f)$,

$$
\begin{gathered}
l_{1}(f)=\operatorname{dist}_{\tilde{H}_{s}^{v}}\left(f, A_{\frac{v}{v}}^{v, 1}\right) ; \\
l_{2}(f)=\inf \left\{\varepsilon>0: \int_{\Omega}\left(\tau_{L_{\varepsilon, s, v}(f)}(y)\right)\left(\Delta^{-\frac{n}{r}}(y)\right) d y<\infty\right\} .
\end{gathered}
$$

Proof. Note that the partial assertions of theorem 4 and theorem 2 can be seen in theorem 3 which we already proved. It remains to show the reverse in these assertions to get sharp theorem. We have the following chain of estimates. We will need some embeddings.

Note first if $\nu>\frac{n}{r}-1 ; 1 \leq p<\infty ; \nu+\frac{n}{r}\left(\frac{1}{p}-1\right)>-1+\frac{n}{r}$

$$
\begin{aligned}
& \int_{\Omega}\left(\int_{R^{n}}|f(x+i y)|^{p} d x\right)^{1 / p} \Delta^{\nu-\frac{n}{r}}(y) d y \leq \\
& \leq c \int_{T_{\Omega}}|f(x+i y)| \Delta^{\nu+\frac{n}{r}\left(\frac{1}{p}-1\right)-\frac{n}{r}}(y) d x d y .
\end{aligned}
$$


Since $A_{\beta}^{p, q}\left(T_{\Omega}\right) \subset A_{\nu}^{s, t}\left(T_{\Omega}\right): 1 \leq p \leq s<\infty, 1 \leq q \leq t<\infty$, $\nu, \beta>\frac{n}{r}-1, \frac{\beta}{q}+\frac{n}{r p}=\frac{\nu}{t}+\frac{n}{r s}$ (see [4]) (in our case $p=q=1 ; s=p$; $\left.t=1 ; \beta+\frac{n}{r}=\nu+\frac{n}{r p} ; \beta=\nu+\frac{n}{r p}-\frac{n}{r}=\nu+\frac{n}{r}(1 / p-1)\right)$ and

$$
A_{\frac{\nu}{v}}^{v, 1} \subset A_{\nu}^{v} ; v \geq 1, \nu \geq v\left(\frac{n}{r}-1\right) .
$$

Remark 2. Approaches we will use in proofs can be applied also for bounded pseudoconvex domains namely unit ball in $C^{n}$. This will extend our previous assertions from [24] where we obtained result for $v=1$ case.

Let us first show if $f$ function has compact support and if $f \in H_{\nu}^{1}$ then we can use Bergman representation (reproducing) formula (for all values of $\nu$ ). Indeed if $f \in H_{\nu}^{1}$ then we use the estimate

$$
\begin{gathered}
\left(\int_{\Omega}\left(\int_{R^{n}}|f(x+i y)| d x\right)^{q} \Delta^{\alpha-\frac{n}{r}}(y) d y\right)^{1 / q} \leq \\
\leq c\left(\sup _{y \in \Omega}\left(\int_{R^{n}}|f(x+i y)| d x\right)^{q}\left(\Delta^{q \nu}(y)\right)\right)^{1 / q} \times \\
\times\left(\int_{\Omega} \Delta^{\alpha-\frac{n}{r}-q \nu}(y) d y\right)^{1 / q} \leq c_{1}\|f\|_{H_{\nu}^{1}}
\end{gathered}
$$

if $\alpha>\frac{n}{r}-1 ; \alpha-\frac{n}{r}-q \nu<-\frac{2 n}{r}+1$ (and similarly $H_{\nu}^{v} \subset A_{\alpha}^{v, q}$ for all $q>q_{0}, \alpha>\frac{n}{r}-1, v \geq 1$ ). This follows from the well-known estimate of integral

$$
I_{\alpha, \beta}(t)=\int_{\Omega} \Delta^{\alpha}(y+t) \Delta^{\beta}(y) d y ; \alpha, \beta \in R
$$

(see [4]). So now we have $H_{\nu}^{1} \subset A_{\alpha}^{1, q}$ if $q$ is large enough $\alpha>\frac{n}{r}-1$ but for all $\tilde{\alpha}>\frac{n}{r}-1, f \in A_{\alpha}^{1, q} ; 1 \leq q<\infty$ (see [19]).

$$
f(z)=\int_{T_{\Omega}}\left(B_{\tilde{\alpha}}(z, w)\right)(f(w))\left(\Delta^{\tilde{\alpha}-\frac{n}{r}}(\operatorname{Im} w)\right) d v(w), z \in T_{\Omega}, \quad\left(K_{1}\right) .
$$

$d v(w)=d u d v, w=u+i v$. This is what we need. (Note similar argument works for $\left.H_{\nu}^{k}, k \geq 1\right)$.

In general case (not only for $f$ functions with compact support as we had above) the mentioned above embeddings between weighted Hardy and Bergman spaces are also true (see [21-22]). The proof is the same 
as in one-dimensional case of analytic functions in the unit disk and it is based on a fact that $L^{p}\left(R^{n}\right)$ norm of $\mathrm{f}$ function is monotone in cone and on existence of $r$-lattice of cone with nice properties (see [21-22]).

As we mentioned a part of theorem $4\left(l_{1} \geq l_{2}\right)$ and first part of theorem $2\left(l_{1} \geq l_{2}\right)$ follows from proof of theorem 3 . The reverse $\left(l_{1} \leq l_{2}\right)$ is similar to proof of theorem 2 for $v=1$, in addition in proof embeddings $\left(A_{1}\right),(\tilde{A})$ should be used. We show now the sharpness (reverse implication $l_{1} \leq l_{2}$ ) in theorems 2 and 4 showing first the basic main case $v=p=1$.

Based on $\left(K_{1}\right)$, we have now that the following decomposition holds $f=f_{1}+f_{2} ; z=x+i y, w=u+i v, z \in T_{\Omega}, w \in T_{\Omega}$

$$
\begin{gathered}
f_{1}(z)=\int_{L_{\varepsilon, \nu}} \int_{R^{n}} B_{\tilde{\alpha}}(z, w) f(w)\left(\Delta^{\tilde{\alpha}-\frac{n}{r}}(\operatorname{Im} w)\right) d v(w) ; \\
f_{2}(z)=\int_{\Omega \backslash L_{\varepsilon, \nu}} \int_{R^{n}} B_{\tilde{\alpha}}(z, w) f(w)\left(\Delta^{\tilde{\alpha}-\frac{n}{r}}(\operatorname{Im} w)\right) d v(w) ; f \in H_{\nu}^{1} .
\end{gathered}
$$

It remains to show that for large enough $\tilde{\alpha}$

$$
\begin{gathered}
\left\|f_{2}\right\|_{H_{\nu}^{1}}<\infty, \\
\left\|f_{1}\right\|_{A_{\nu}^{1}}<\infty .
\end{gathered}
$$

This gives what we need (sharpness of theorem 2 and half of theorem 4 for $v=p=1$ ).

We have

$$
\operatorname{dist}_{H_{\nu}^{1}}\left(f, A_{\nu}^{1}\right)=\inf _{g \in A_{\nu}^{1}}\|f-g\|_{H_{\nu}^{1}} \leq c\left\|f-f_{1}\right\|_{H_{\nu}^{1}}=\left\|f_{2}\right\|_{H_{\nu}^{1}}<\infty
$$

To show (9) we have to use the well known estimates for $\Delta^{t}, B_{\alpha}$. We have now by Fubbini's theorem

$$
\begin{gathered}
\int_{R^{n}}\left|f_{2}(x+i y)\right| d x \Delta^{\nu}(y) \leq \\
\leq \int_{R^{n}} \Delta^{\nu}(y) \int_{\Omega \backslash L} \int_{R^{n}}\left|B_{\tilde{\alpha}}(z, w)\right||f(w)| \Delta^{\tilde{\alpha}-\frac{n}{r}}(\operatorname{Im} w) d v(w) d x \leq \\
\leq c\left(\Delta^{\nu}(y) \int_{R^{n}} \int_{\Omega \backslash L}\right) \int_{R^{n}}\left|B_{\tilde{\alpha}}(z, w)\right||f(w)| \Delta^{\tilde{\alpha}-\frac{n}{r}}(\operatorname{Im} w) d v(w) d x \leq
\end{gathered}
$$




$$
\begin{gathered}
\leq c \Delta^{\nu}(y) \int_{\Omega \backslash L} \int_{R^{n}}|f(w)| \Delta^{\tilde{\alpha}-\frac{n}{r}}(v) \Delta^{-\tilde{\alpha}}(v+y) d v(w) \leq \\
\leq \varepsilon c \Delta^{\nu}(y) \int_{\Omega} \Delta^{-\tilde{\alpha}}(v+y) \Delta^{\tilde{\alpha}-\frac{n}{r}-\nu}(v) d v \leq \varepsilon c ; \\
\tilde{\alpha}>\frac{n}{r}-1 ; \nu>\frac{n}{r}-1 ; w=u+i v, z=x+i y .
\end{gathered}
$$

To show (10) (and then based on $\left(A_{1}\right)$ theorem 4 (part 1$)$ ) we have to use the following estimates

$\int_{T_{\Omega}}\left|f_{1}(x+i y)\right| \Delta^{\nu-\frac{n}{r}}(y) d x d y \leq c\|f\|_{H_{\nu}^{1}} \int_{L_{\varepsilon, \nu}(f)} \frac{\Delta^{\tilde{\alpha}-\frac{n}{r}-\nu}(v)}{\Delta^{\tilde{\alpha}-\nu}(v)} d v \leq c\|f\|_{H_{\nu}^{1}}$.

To set these estimates note that (see [1])

$$
\begin{gathered}
\int_{T_{\Omega}}\left|B_{\tilde{\alpha}}(z, w)\right| d v_{\bar{\nu}}(z)=\int_{T_{\Omega}}\left|B_{\tilde{\alpha}}(z, w)\right| \Delta^{\nu-\frac{n}{r}}(y) d x d y \leq c \Delta^{-(\tilde{\alpha}-\nu)}(v) \\
d v_{\bar{\nu}}=\left(\Delta^{\bar{\nu}}(y)\right) d x d y ; \bar{\nu}=\nu-\frac{n}{r}, w=u+i v, z=x+i y
\end{gathered}
$$

see also [4]; for large enough $\tilde{\alpha}$. Theorems 2 and 4 for $v=1=s=p$ are proved. Using $\left(A_{1}\right)$ Theorem 4.1) is proved also.

Remark 3. We can use $A_{s}^{p} \subset A_{\nu}^{\tilde{p}, q}$ to set various not sharp results too.

Proof. (general case). The proof of sharpness of theorem 2 (general case) is now a modification of proof of particular case we had above. First we have to show that if $f \in H_{s}^{v}$ then for large enough $\tilde{\alpha}, \tilde{\alpha}>\alpha_{0}$

$$
f(z)=\int_{\Omega} \int_{R^{n}} B_{\tilde{\alpha}}(z, w) f(w) \Delta^{\tilde{\alpha}-\frac{n}{r}}(\operatorname{Im} w) d v(w) .
$$

For this we have to use also a known fact that (see [19])

$$
f(z)=\int_{T_{\Omega}} B_{\tilde{\alpha}}(z, w) f(w) \Delta^{\tilde{\alpha}-\frac{n}{r}}(\operatorname{Im} w) d v(w),
$$

for all $\tilde{\alpha}$ if $\tilde{\alpha}>\frac{n}{r}-1 ; f \in A_{\nu}^{p, q} ; 1 \leq p<\infty$; and if $1 \leq q<\nu+\frac{n}{r}-1=\tilde{q}_{\nu, p}$; or $\left(\tilde{q}_{\nu, p}\right)=\infty$; if $\frac{n}{r} \leq p^{\prime} ; \frac{1}{p}+\frac{1}{p^{\prime}}=1, \nu>\frac{n}{r}-1$.

Under condition $\frac{n}{r}<p^{\prime}$ we put $v=p$ and our estimate (B) set what we need for any $f$ function with compact support. Following the [4] (proof of 
theorem 4.10) and previous theorem we have to show $\left(f_{1}\right.$ and $f_{2}$ defined above) $\left\|f_{2}\right\|_{H_{s}^{v}}<\varepsilon c_{1}$ and $\left\|f_{1}\right\|_{A_{\nu}^{v}}<c\|f\|_{H_{s}^{v}} ; \frac{n}{r}<v^{\prime} ; \nu>\frac{n}{r}-1 ; s=\frac{\nu}{v}$; $1 \leq v<\infty, \frac{1}{v^{\prime}}+\frac{1}{v}=1$.

Note these estimates for $v=1$ are obtained previously in [23]. We are now using Minkowski and Young's inequality

$$
\begin{gathered}
\left\|f_{2}\right\|_{H_{s}^{v}} \leq c \sup _{y}\left[\Delta^{s}(y)\right] \times \\
\times\left(\int_{R^{n}}\left(\int_{\Omega \backslash L_{\varepsilon}} \int_{R^{n}}\left|B_{\tilde{\alpha}}(z, w) \| f(w)\right|\left[\Delta^{\tilde{\alpha}-\frac{n}{r}}(\operatorname{Im} w)\right] d v(w)\right)^{v} d x\right)^{\frac{1}{v}} \leq \\
\leq c \sup _{y}\left[\Delta^{s}(y)\right] \int_{\Omega \backslash L_{\varepsilon}}\left[\Delta^{-\tilde{\alpha}}(\operatorname{Im} z+\operatorname{Im} w)\right]\left[\|f(w)\|_{L^{v}}\right] \Delta^{\tilde{\alpha}-\frac{n}{r}}(\operatorname{Im} w) d \tilde{v} \leq \\
\leq \varepsilon c \sup _{y}\left(\Delta^{s}(y)\right)\left(\int_{\Omega} \Delta^{-\tilde{\alpha}}(x+y) \Delta^{\tilde{\alpha}-\frac{n}{r}-s}(x) d x\right) \leq c \varepsilon ; \tilde{v}=\operatorname{Im} w ;
\end{gathered}
$$

Using $(\tilde{A})$ we also have

$$
\begin{aligned}
\left\|f_{1}\right\|_{A_{\nu}^{v}} \leq & c \int_{\Omega} \Delta^{\frac{\nu}{v}-\frac{n}{r}}(\tilde{y})\left(\int_{R^{n}}\left|f_{1}(x+\tilde{y} i)\right|^{v} d x\right)^{\frac{1}{v}} d \tilde{y} \leq \int_{\Omega}\left[\Delta^{\frac{\nu}{v}-\frac{n}{r}}(\tilde{y})\right] \times \\
& \times \int_{L_{\varepsilon}} \Delta^{-\tilde{\alpha}}(\tilde{y}+y)\left\|f_{y}\right\|_{L^{v}} \Delta^{\tilde{\alpha}-\frac{n}{r}}(y) d y d \tilde{y} \leq c_{1}\|f\|_{H_{s}^{v} \times} \\
\times & \int_{L_{\varepsilon}} \int_{\Omega} \Delta^{\frac{\nu}{v}-\frac{n}{r}}(\tilde{y}) \Delta^{-\tilde{\alpha}}(\tilde{y}+y) d \tilde{y} \Delta^{\tilde{\alpha}-\frac{n}{r}-s}(y) d y \leq c_{2}\|f\|_{H_{s}^{v}} ; \nu>\left(\frac{n}{r}-1\right) v ;
\end{aligned}
$$

$\tilde{\alpha}>\alpha_{0}$;

We above used Minkowski and then Young's inequality. Theorem 2 is proved.

We show now the sharpness in theorem 4 (part 3). The proof is very similar to proofs of previous assertion we just finished and careful analysis of that proof shows it is actually contained there. Theorem is proved.

\section{$\S 3$. On new estimates for $\operatorname{dist}_{A_{\alpha}^{\infty}}\left(f, A_{\nu}^{q, p}\right)$}

Here we provide another sharp theorem for dist function and add some vital related remarks.

In our previous theorem 1 we solved $\operatorname{dist}_{A_{\alpha}^{\infty}}\left(f, A_{\nu}^{q}\right)$ problem based on embedding $A_{\nu}^{q} \subset A_{\alpha}^{\infty}$; for certain fixed $\nu ; 1 \leq q<\infty$ where $A_{\nu}^{q}=A_{\nu}^{q, q}$, $A_{\alpha}^{\infty}=\left\{f \in H\left(T_{\Omega}\right): \sup _{z \in T_{\Omega}}|f(x+i y)| \Delta^{\alpha}(y)<\infty\right\} ; \alpha \geq 0, z=x+i y$. 
We generalize this result to $A_{\alpha}^{p, q}$. The distance problem still can be posed since we have an embedding

$$
\sup _{z \in T_{\Omega}}|F(z)| \Delta^{\frac{n}{r p}+\frac{\nu}{q}}(y) \leq c\|F\|_{A_{\nu}^{p, q}, p, q \in[1, \infty) ; \nu>\frac{n}{r}-1}
$$

(see [4], [23]).

So we can pose a dist problem based on this inclusion.

Remark 4. Note that if $\left\{B_{j}\right\}$ is an r-lattice in tubular domain $T_{\Omega}$ then we have for all $p \in(0, \infty), \nu>\frac{n}{r}-1$

$$
\sum_{j \geq 0} \Delta^{\nu-\frac{n}{r}}\left(\zeta_{i}\right) \int_{B_{j}}|g(\zeta)|^{p} d \zeta \leq\|g\|_{A_{\nu}^{p, p}}^{p} \leq c \sum_{j \geq 0} \Delta^{\nu-\frac{n}{r}}\left(\zeta_{i}\right) \int_{B_{j}}|g|^{p} d \zeta
$$

(see [4], [23]).

We define a space $S_{\alpha}^{p, q}$ as a space of analytic functions with norms

$$
\sum_{j} \Delta^{\alpha}\left(\zeta_{j}\right)\left(\int_{B_{j}}|g(\zeta)|^{p} d \zeta\right)^{\frac{q}{p}} ; 1 \leq p, q<\infty, \alpha>0
$$

and using for fixed $\nu, t$ the fact that $A_{\nu}^{p, p} \subset A_{t}^{p, q}, p \leq q$ (see above) we can pose various other dist problems based on these inclusions. Note we have also similarly, (see [4])

$$
\|F\|_{A_{\nu}^{p, q}} \asymp\left(\sum_{j} \Delta^{\nu}\left(y_{j}\right)\left\|F\left(\circ+i y_{j}\right)\right\|_{p}^{q}\right)^{\frac{1}{q}} ; 1 \leq p, q<\infty, \nu>\frac{n}{r}-1,
$$

for $\left\{y_{j}\right\}$ lattice in symmetric cone $\Omega$, where the inner norm is the norm of Hardy space. We can then define similarly $M_{\nu}^{p, q}$ classes. From here for example various problems of estimates of $\operatorname{dist}_{S_{\alpha}^{p, q}}\left(f, A_{t}^{v, s}\right)$ or $\operatorname{dist}_{A_{\alpha}^{p, q}}\left(f, S_{t}^{v, s}\right)$ (or with $M$ instead of $S$ ) functions with some restrictions on $v, s, p, q, t, \alpha$ arise naturally. This will be the base of our further work in this direction.

The following theorem for classical Bergman $A_{\nu}^{p}$ spaces has been proved above. We formulate it again for readers convenience.

Theorem A. Let $1 \leq p<\infty, \nu>p\left(\frac{n}{r}-1\right) ; \beta_{0}>t+\frac{n}{r}-1 ; t=$ $=\frac{1}{p}\left(\nu+\frac{n}{r}\right)$. Let $f \in A_{t}^{\infty}$. Then for all $\beta, \beta>\beta_{0}$

$$
l_{1}=\operatorname{dist}_{A_{t}^{\infty}}\left(f, A_{\nu}^{p}\right) \asymp
$$


$\asymp \inf \left\{\varepsilon>0: \int_{T_{\Omega}}\left(\int_{V_{\varepsilon, t}(f)} \frac{\Delta^{\beta-t-\frac{n}{r}}(\operatorname{Im} z)}{\Delta^{\beta+\frac{n}{r}}\left(\frac{z-\bar{w}}{i}\right)} d u d v\right)^{p} \Delta^{\nu-\frac{n}{r}}(y) d x d y<\infty\right\}=l_{2}(f)$,

where $V_{\varepsilon, t}(f)=\left\{z \in T_{\Omega}:|f(z)| \Delta^{t}(\operatorname{Im} z) \geq \varepsilon\right\}$.

We generalize this theorem to mixed norm $A_{\nu}^{p, q}$ spaces below.

Let $B_{\beta, \mu}=\frac{\mu+\frac{n}{r}-1}{\beta} ; A_{\beta, \mu}=\frac{\mu-\left(\frac{n}{r}-1\right)}{\beta-\left(\frac{n}{r}-1\right)}$.

Theorem 5. Let $\beta_{0}>t+\frac{n}{r}-1 ; t=\frac{n}{r s}+\frac{\mu}{q} ; f \in A_{t}^{\infty}$. Let also $\mu>\frac{n}{r}-1$, $s, q \in[1, \infty) ; \beta_{0}>\frac{n}{r}-1 ; q<\frac{\mu+\frac{n}{r}-1}{\frac{n}{r}-1}$. Let also

$V_{\varepsilon, t}=\left\{z \in T_{\Omega}:|f(z)| \Delta^{t}(\operatorname{Im} z) \geq \varepsilon\right\} ; z=x+i y ; w=u+i v$. Then for all $\beta>\beta_{0}$, we have

$$
\asymp \inf \left\{\varepsilon>0: \int_{\Omega}\left(\int_{R^{n}}\left(\int_{V_{\varepsilon, t}(f)} \frac{\Delta^{\beta-t-\frac{n}{r}}(\operatorname{Im} z) d x d y}{\Delta^{\beta+\frac{n}{r}}\left(\frac{z-\bar{w}}{i}\right)}\right)^{s} d u\right)^{\frac{q}{s}} \Delta^{\mu-\frac{n}{r}}(v) d v<\infty\right\} .
$$

The proof of this theorem follows immediately from arguments of proof of previous theorem 1 and the theorem below on boundedness of Bergman projection in mixed norm spaces with positive Bergman kernel. Note also that the half of theorem (one implication) is immediately follows from the arguments of the proof of theorem 1 .

Theorem 6. (see [19]). Let $\mu, \nu \in R, 1 \leq p, q<\infty$, let also

$$
P_{\mu}^{+} f(z)=\int_{T_{\Omega}} \frac{|f(w)| \Delta^{\mu-\frac{n}{r}}(\operatorname{Im} w) d v(w)}{\left|\Delta^{\mu+\frac{n}{r}}\left(\frac{z-\bar{w}}{i}\right)\right|}
$$

then $P_{\mu}^{+}$is bounded on $\left(L_{\nu}^{p, q}\right)\left(T_{\Omega}\right) ; \mu, \nu>\frac{n}{r}-1$; if and only if

$$
\max (A, B) \leq q<\frac{\nu+\frac{n}{r}-1}{\frac{n}{r}-1} ; B=B_{\mu, \nu}, A=A_{\mu, \nu}
$$

We omit details leaving them to readers. The proof will be given in our another paper. 
Remark 5. Some results of this paper have also direct analogues for harmonic function spaces with mixed norm of several variables in unit ball and upper half spaces, see also [1], [25] for related results in particular values of parameters.

This work partially was done by authors during the visit of first author to IMAR (Romania) at the end of 2012. The author thanks IMAR for support.

\section{References}

[1] Arsenović M., Shamoyan R. On some extremal problems in spaces of harmonic functions. ROMAI Journal, 2011, vol. 7, pp. 13-34.

[2] Shamoyan R. F., Mihić O. On new estimates for distances in analytic function spaces in the unit disc, polydisc and unit ball. Bollet. de la Asoc. Matematica Venezolana, 2010, vol. 42, no. 2, pp. 89-103.

[3] Shamoyan R., Mihić O. On new estimates for distances in analytic function spaces in higher dimension. Siberian Electronic Mathematical Reports, 2009, vol. 6, pp. 514-517.

[4] Bekolle D., Bonami A., Garrigos G. and others. Lecture notes on Bergman projectors in tube domains over cones. Yaounde: Procedings of the international Workshop on classical Analysis, 2001, 75 p.

[5] Shamoyan R., Arsenovic M. Some remarks on extremal problems in weighted Bergman spaces of analytic functions. Comm. Korean Math. Society, 2012, vol. 6 .

[6] Zhao R. Distance from Bloch functions to some Möbius invariant spaces. Ann. Acad. Sci. Fenn., 2008, vol. 33, pp. 303-313.

[7] Shamoyan R., Radnia M. Some new estimates for distances in analytic function spaces of several complex variables and double Bergman representation formula. Journal of Nonlinear Sci. and Applications, 2010, vol. 3, no. 1 , pp. $48-54$.

[8] Xu W. Distances from Bloch functions to some Mobious invariant function spaces. Journal of function spaces and applications, 2009, vol. 7, pp. 91-104.

[9] Anderson J. M., Clunie J., Pommerenke Ch. On Bloch functions and normal functions. Journal. Reine. Angew. Math, 1974, vol. 270, pp. 12-37.

[10] Anderson J. M. Bloch functions - the basic theory. Operators and Function theory. Lancaster, Reidel, Dordrecht, 1985, vol. 153, pp. 1-17.

[11] Xiao J. Geometric $Q_{p}$ functions. Frontiers in Mathematics, BirkhauserVerlag, 2006. 
[12] Ghatage P., Zheng D. Analytic functions of bounded mean oscillation and the Bloch space. Integ. Equat. Oper. Theory, 1993, vol. 17, pp. 501-515.

[13] Ahlfors L. Bounded analytic functions. Duke Math. Journal, 1947, vol. 14, pp. 1-14.

[14] Khavinson S. On an extremal problem in the theory of analytic function. Russian. Math. Survey, 1949, vol. 4 (32), pp. 158-159.

[15] Rudin W. Analytic functions of Hardy class. Trans. Amer. Math. Soc., 1955, vol. 78, pp. 46-66.

[16] Khavinson D., Stessin M. Certain linear extremal problems in Bergman spaces of analytic functions. Indiana Univ. Math. Journal, 1997, vol. 3, pp. 46.

[17] Debertol D. Besov spaces and boundedness of weighted Bergman projections over symmetric tube domains. Dottorato di Ricerca in Matematica, Universita di Genova, Politecnico di Torino (April), 2003.

[18] Faraut J. and Koranyi A. Analysis on symmetric cones. Oxford Mathematical Monographs, Oxford Science Publications, The Clarendon Press, Oxford University Press, New York, 1994, XII, 382 p. ISBN:0-19-853477-9.

[19] Sehba B. Bergman-type integral operators in tube domains over symmetric cones. Proceedings of Edinburg Math. Soc.

[20] Duren P. and Schuster A. Bergman spaces. Mathematical Surveys and Monographs, 2004, vol. 100. AMS, RI.

[21] Bekolle D., Bonami A., Garrigos G., Ricci F., Sehba B. Analytic Besov spaces and Hardy type inequalities in tube domains over symmetric cones. Jour. fur. reine und ang., 2010, vol. 647, pp. 25-56.

[22] Bekolle D., Bonami A., Garrigos G., Ricci F. Littlewood-PAley decomposition and Besov spaces related to symmetric cones. Preprint, 2008, arxiv.

[23] Shamoyan R. On an external problem in tubular domains over symmetric cones. Preprint, 2012, arxiv.

[24] Shamoyan R., Mihic O. New distance estimates in spaces of analytic functions in unit ball and polydisk. Bulletin Mat Society Venezolana, 2010, vol. 42 , pp. $89-102$.

[25] Shamoyan R. F. On some extremal problems in certain harmonic function spaces of several variables related to mixed norm spaces // Issues of Analysis, 2013, vol. 2 (20), no. 1, pp. 43-58.

The work is received on September 23, 2013.

Bryansk State Technical University,

Bulvar 50-letiya Oktyabrya, 7, 241035 Bryansk, Russia.

E-mail: rshamoyan@gmail.com, SergKurilenko@gmail.com 